\title{
Management of White Spot Lesions Using Resin Infiltration Technique: A Review
}

\author{
Zafer Azizi \\ Pediatric Dentistry Department, Arab American University, Palestine
}

Copyright (C) 2015 Horizon Research Publishing All rights reserved.

\begin{abstract}
Management of white spot lesions has always been an important issue in modern dentistry. Many preventive measures have been suggested recently to slow the demineralization process and allow better remineralization of enamel as soon as white spot lesions begin to appear and just before proceeding into the cavitated lesion stage. A new technology in dealing with such lesions is known as "Resin Infiltration". It seems to provide an intermediary treatment modality between prevention and restorative therapy. The concept was introduced as a micro-invasive approach for the management of smooth surface and proximal non-cavitated carious lesions. The purpose of this review is to present the scientific basis and principles of the usage of resin infiltration technique as well as its importance in the clinical practice.
\end{abstract}

Keywords Resin Infiltration Technique, ICON, White Spot Lesions, Smooth Surface Lesions, Non-cavitated Lesions, Proximal Lesions

\section{Introduction}

Enamel demineralization and remineralization are considered as a dynamic and continuous process that occurs throughout the whole life of a tooth. White spot lesions \& early enamel lesions are probably considered as a first sign of dental caries disease. They can occur on any tooth surface in the oral cavity where the microbial biofilm is allowed to develop and remain for a period of time. These areas of demineralized enamel that usually develop because of prolonged plaque accumulation would lose their translucency because of an extensive subsurface porosity caused by demineralization. If the process is not interrupted and demineralization reversed, they might progress from demineralization, to non-cavitated lesions, and finally to cavitated lesions.

With the evolution of dentistry and the progress in preventive and adhesive technologies, new techniques are applied for preventing and stopping the carious process in the aim to preserve the tooth structure: so called "non invasive" and "minimal invasive dentistry". Minimal invasive dentistry is "a systematic respect for the original tissue" by removing and replacing with as little tissue loss as possible. $^{(1)}$

Because of the absence of any restorative material which can fully replace the enamel and dentin, preservation of tooth structure should be the main concern in any treatment plan. After the principle of extension for prevention by Black, new minimal intervention treatments were introduced, respecting the dental structures and aiming to preserve the tooth integrity and minimize treatment side-effects as much as possible.(2)

The Concept of minimally invasive techniques in dentistry requires a good diagnosis of risk and lesions, the use of the right preventive treatment to stop disease, the restorations of the lesions with as little healthy tissue loss as possible, the use of durable materials and the prevention of recurring disease.(3)

Treatment of proximal lesions varies from non-invasive/preventive to invasive operative procedures. The choice of a treatment option depends on the extent of the lesion as assessed by radiographs. Lesions confined to the outer half of the enamel are rarely cavitated and are managed with improvement of patient's oral hygiene and dietary control in addition to remineralization. Clinically, the degree of remineralization seems to be limited, and this has been attributed to the presence of organic substances attached to the enamel surface occluding the underlying pores in the carious lesions. ${ }^{(4)}$ In addition, the depth of remineralization is limited resulting in a slow, but continuous progression of the lesion into deeper layers. ${ }^{(4)}$ Sealing of interproximal initial lesions has also been suggested, but despite its ability to arrest some caries lesions, it still exhibited a high percentage of lesion progression. ${ }^{(5)}$ In addition, the technique is difficult clinically. It only seals the superficial area of the lesion and the excess resin cannot be easily removed promoting the development of secondary caries and periodontal irritation. ${ }^{(6)}$

Once the lesions progress into the middle or inner third of dentine, they are cavitated and invasive restorative treatment is recommended. Uncertainty in treatment often exists for radiolucencies extending into the inner part of enamel or the 
outer third of dentine. In a clinical study, $8-11 \%$ and $22-44 \%$ of such lesions respectively were found to be cavitated. ${ }^{(7)}$ The treatment decision is difficult in those cases because of the risk of under- or over-treatment.

\section{Resin Infiltration Concept}

Caries infiltration is described as a micro-invasive technology that fills, reinforces, and stabilizes demineralized enamel without drilling or sacrificing healthy tooth structure. The micro-invasive infiltration can be used to treat smooth surface and proximal carious lesions up to the first third of dentin (D-1).It prevents lesion progression and increases life expectancy of a tooth. It provides an alternative to microabrasion and other restorative treatments for cariogenic white spot lesions. Once infiltrated, white spot lesions take on the appearance of the surrounding healthy enamel.

Resin infiltration technique is a novel technology that seems to bridge the gap between noninvasive and minimally invasive treatment of initial dental caries, postponing as long as possible the need for a restoration. ${ }^{(\mathbf{8})}$

The concept of caries infiltration was first developed at the Charité Berlin and the University of Kiel as a micro-invasive approach for the management of smooth surface and proximal non-cavitated caries lesions. It is marketed under the name $\operatorname{Icon}^{\circledR}(D M G$ America Company, Englewood, $N J) .{ }^{(9)}$

The principal of resin infiltration is to perfuse the porous enamel with resin by capillary action. This aims to arrest lesion progression by occluding the microporosities that provide diffusion pathways for the acids and dissolved materials. ${ }^{(10)}$ It has been debated that bacteria entrapped at the base of lesions could trigger and spread the caries process. It has been well established that the count of bacteria in non-cavitated lesions is low and not detrimental especially if properly sealed. ${ }^{(\mathbf{1 0})}$

\section{Resin Infiltration Steps and Procedure}

Despite the novelty of the resin infiltration technique, several studies were conducted in vitro and in vivo that tested the effect of different etching agents on the enamel surface of the initial caries lesions and the subsequent penetration ability of different adhesive systems. ${ }^{(11-15)}$ The results advocate the use of $15 \%$ hydrochloric acid gel for 2 minutes, followed by drying of the surface and application of a low viscosity resin of type TEGDMA (tri-ethylene glycol dimethacrylate).The procedure for resin infiltration is fairly simple and acceptable by operators and patients. ${ }^{(16)} \mathrm{ICON}^{\circledR}$ is marketed in two different forms: Proximal surface and vestibular surface kits. The principle of usage in both is similar except for the need for separation in case of proximal lesion treatment. ${ }^{(\mathbf{9}, \mathbf{1 7})}$ As the mineralized surface layer of the non-cavitated lesions hampers resin from penetrating into the lesion. This layer should be removed. Hydrochloric acid gel (15\%) has been demonstrated to be superior to $37 \%$ phosphoric acid gel in removing the surface layer of natural enamel lesions when applied for 120 seconds. ${ }^{(11,12,18,19)}$

Researchers reported that etching with phosphoric acid affected only the outermost $25 \mu \mathrm{m}$ of the surface. ${ }^{(\mathbf{2 0})}$ However, superficial phosphoric acid etching causes the caries lesion to extend into the deeper enamel layers resulting in probable cavitation in the future. ${ }^{(20)}$ The penetration depth of $15 \%$ HCL etching is more than twice $(58 \mu \mathrm{m})$ that of phosphoric acid, enabling penetration into the deepest part of the lesion and eliminating the decalcified areas, preventing further attacks. ${ }^{(13)}$ The surface is then dehydrated with $99 \%$ ethanol (ICON Dry) to facilitate the drying process. The philosophy of using ethanol wet bonding technique, in a clinically acceptable dehydration period, is based on the assumption that it will coax hydrophobic monomers to infiltrate into demineralized wet enamel or dentine, and improve the efficacy of penetration of the hydrophobic infiltrate (TEGDMA) to get a well-defined, resin-infiltrated layer. ${ }^{(21,22)}$

In the water wet-bonding technique, the presence of water in the demineralized dentin after phosphoric acid etching is essential to prevent the collapse of the dentin matrix. The space that is occupied by the water surrounding the non-collapsed collagen fibrils can then be infiltrated by the resin monomers to form the hybrid layer. ${ }^{(\mathbf{2 1 , 2 2})}$ However, the presence of residual water in the current wet-bonding technique is a potential factor for phase separation of the hydrophobic monomers, which have limited water solubility. Recent studies have shown that it is possible to bond hydrophobic resin monomers to acid-etched dentin and enamel with a new technique called "ethanol wet bonding". ${ }^{(21,22)}$ The technique involves slowly replacing water within the demineralized collagen matrix with ascending concentrations of ethanol, allowing the latter to penetrate the collagen matrix without causing additional shrinkage of the interfibrillar spaces thus preventing phase separation of hydrophobic resin monomers.

The last step involves light curing of the resin following a three minute application time. A repeated application for another one minute is performed then the resin is light cured again. The excess resin is then removed and the surface is polished. ${ }^{(17)}$ Several studies on artificial caries-like lesions have demonstrated that commercially available adhesives having infiltrated the micropores of demineralized areas revealed a considerable reduction of lesion progression by either double application or extended penetration times. However, using resin infiltrants have proved superior penetration potential, depth and arresting caries progression capacity. ${ }^{(13,23-26)}$

Using resins with high penetration coefficients $(>200 \mathrm{~cm} / \mathrm{sec})$ showed superior ability to penetrate natural lesions compared with resins with lower penetration coefficient. $^{(\mathbf{1 3 , 1 8 , 2 5})}$ Using infiltrants with a refractive index closer to that of hydroxyapatite improved the masking effect 
of the white spot lesions. ${ }^{(27)}$ The resin is applied twice because of the shrinkage of the material after the first application resulting in the generation of space that can be then occluded by a second application. ${ }^{(\mathbf{2 8 , 2 9})}$ Polishing of the infiltrated lesion was found to improve the stability of the masking effect most likely due to reduction in surface porosity and possible removal of the oxygen inhibition layer. ${ }^{(30)}$

\section{Treatment of White Spot Lesions with Resin Infiltration Technique Proximal Lesions}

Caries infiltration technique offers a microinvasive option for the management of proximal lesions that are confined to the outer and inner enamel and to the outer third of dentine as diagnosed by radiographs. Caries infiltration technique aims to arrest the lesion progression by occluding the microporosities that provide diffusion pathways by acids and dissolved minerals. ${ }^{(10)}$ The resin infiltrate exhibits a very low viscosity, low contact angles to the enamel, and high surface tension to allow complete penetration of the resin into the lesion body of the enamel caries. ${ }^{(25)}$

Paris et al. (2011)(7) attempted to test the infiltration pattern in cavitated and non-cavitated proximal lesions. It was concluded that under in vitro conditions, the infiltrant penetrates in most parts of the demineralized enamel but is not capable of filling up cavities and therefore the efficacy of the resin infiltration technique, particularly in lesions with larger cavitations, might be impaired.(7)Moreover, the infiltrated artificial lesions showed increased resistance to demineralization and arrested progression of caries lesions both in vitro and in situ. $(15,31,32)$

Clinical trials have shown that for non-cavitated proximal caries lesions being extended radiographically -at maximumto the outer third of dentine, caries infiltration, in combination with non-operative procedures, were significantly more efficient in arresting lesion progression compared with non-operative measures alone.(33)

Paris et al. (2010) ${ }^{(34)}$ conducted a randomized split mouth placebo-controlled clinical trial to assess proximal lesions progression in 22 young adults using digital substraction radiography technique. Within the follow up period of 18 months, caries infiltration has reduced lesion progression in non-cavitated interproximal lesions extending up to the outer third of dentine. ${ }^{(34)}$ More recently, Martignon et al. $(2012)^{(35)}$ conducted a randomized split-mouth controlled clinical trial aiming to assess the therapeutic effects of infiltration versus sealing for controlling interproximal lesion progression. The study was conducted on 39 adults, each with 3 proximal surface lesions identified radiographically. The lesions were randomly allocated to resin infiltration, sealing or placebo treatment. The lesions were followed up for 3 years and caries progression was assessed using digital substraction imaging. Both resin infiltration and sealing were able to stop the progression of caries with no significant differences between the two. ${ }^{(35)}$

\section{Resin Infiltration in Primary Teeth}

The use of resin infiltration technique for the management of non-cavitated caries lesions in primary teeth is expected to differ from that in permanent teeth. Firstly, the progression rate of proximal caries lesions in primary molars is significantly higher than in permanent teeth. ${ }^{(36,37)}$ Secondly, the ultrastructure and mineral composition of primary enamel is different; as it is less mineralized and more porous and aprismatic in comparison with permanent enamel. ${ }^{(38)}$ As a result, the diffusion coefficient seems to be greater in primary enamel. Thirdly, the proximal surface layer is less mineralized and thinner in primary molars compared with permanent ones. ${ }^{(38)}$

In consequence, etching the surface of primary teeth with $15 \%$ hydrochloric acid for 120 seconds resulted in reliable and considerable erosion of the mineralized surface layer deeper than that seen in permanent teeth. ${ }^{(\mathbf{1 4})}$ Primary teeth exhibited better infiltrant penetration than permanent teeth even after shorter duration of application. In fact, after 1 minute of application of the resin on primary molars in vitro, the non-cavitated lesions were deeply and consistently infiltrated. ${ }^{(39,40)}$ On the other hand, 3-5 minutes are required to almost completely infiltrate a natural lesion in permanent teeth with an extension into the inner half of enamel. In fact, irrespective of the lesion depth, a 1 minute application would result in rather superficial infiltration only in permanent teeth. ${ }^{(41)}$ Following 5 minutes resin application, Liu et al. $(2013)^{(24)}$ found no significant differences in the overall penetration between primary and permanent molar lesions except in lesions extending to the outer half of enamel. In those lesions, the penetration ability of the resin was significantly higher in primary molars. ${ }^{(24)}$

Clinical trials have shown that for non-cavitated proximal caries lesions being extended radiographically -at maximumto the outer third of dentine, caries infiltration, in combination with non-operative procedures, were significantly more efficient in arresting lesion progression compared with non-operative measures alone in primary molars.(42)

Ekstrand et al. $(2010)^{(42)}$ conducted a split-mouth study to assess the efficacy of resin-infiltrated lesions covered by fluoride varnish (FV) versus FV treatment only of proximal lesions on deciduous molar teeth. The study lasted for 1 year and it included 50 children with moderate to high caries risk, with 2 or more superficial proximal lesions on deciduous molar teeth detected on bitewing radiographs. The lesions were randomly allocated to the two treatment groups. Lesion progression was assessed clinically and radiographically. Proximal caries in primary molars treated by resin infiltration and fluoride varnish progressed significantly less $(23 \%)$ than those treated with fluoride varnish only $(61 \%)$ after one year. The difference in clinical and radiographic therapeutic effects of both resin infiltration with FV over FV 
alone was $>35 \%{ }^{(42)}$

\section{Smooth Surface and Post-orthodontic Demineralization}

The innovative idea of using the resin infiltration technique in the management of white spot smooth surface lesions was based on the side effect caused by the treatment actually masking these early lesions. The ideal treatment of non-cavitated carious lesions (white spot lesions) should arrest the lesion progression and improve the esthetics.

In terms of esthetics, masking enamel lesions by resin infiltration is based on changes in light scattering within the lesions. Sound enamel has refractive index (RI) of 1.62. The microporosities of enamel carious lesions are filled with either a watery medium (RI 1.33) or air (RI 1.0). ${ }^{(23)}$ The difference in refractive indices between the enamel crystals and medium inside the porosities causes light scattering that results in a whitish opaque appearance of these lesions, especially when they are desiccated. ${ }^{(43)}$ The novel technique used involves the infiltration of the carious lesions with resin (RI 1.52) that, in contrast to the watery medium, cannot evaporate and is similar to that of apatite crystals. This makes the difference in refractive indices between porosities and enamel to be negligible and lesions appear similar to the surrounding sound enamel. Lesions lose their whitish opaque color and blend reasonably well with surrounding natural tooth structure. ${ }^{(43)}$

Resin infiltration technique was found to be an effective treatment for masking white spot lesions in vitro and in vivo. It is believed that the masking effect depends on the lesion depth and activity.

A recent in vitro study showed superior esthetic results of resin infiltration of artificial white spots when compared with remineralization after the application of fluoride. ${ }^{(44)}$ Paris et al. (2013) ${ }^{(27)}$ assessed the masking effect of resin infiltration in artificially induced white spot lesions in bovine teeth using different infiltrate materials. Not only the lesions were masked and significantly had better color match with normal enamel but also they resisted discoloration following treatment better than the normal enamel. ${ }^{(27)}$

Paris and Meyer-Lueckel ${ }^{(23)}$ reported the first successful immediate improvement of esthetic appearance of white spot lesions that remained stable until the $\mathbf{1 0}^{\text {th }}$ month of follow up. ${ }^{(23)}$ Shivanna and Shiivakumar $(2011)^{(45)}$, and Basaran et al. $(2011)^{(20)}$ reported the successful immediate masking of post-orthodontic lesions in teeth treated with resin infiltration technique. Shivanna and Shiivakumar $(2011)^{(45)}$ reported result stability at the end of a 3-month review period.

A first clinical study demonstrated successful masking of post-orthodontic lesions (61\% completely and 33\% partially masked lesions) with resin infiltration. ${ }^{(28)}$

In China, Feng $(2013)^{(46)}$ evaluated clinically the effectiveness of resin infiltration in masking post-orthodontic white spots in eight patients with 6 maxillary anterior teeth showing signs of decalcification (total 48 teeth) using standardized digital photography taken before, immediately after, as well as 1 week, 6 and 12 months after treatment. ${ }^{(46)}$ The images were analyzed using image analysis software and the size of the white spot lesion and the whole tooth facial surface were measured. Among the 48 teeth, 11 teeth $(22.9 \%)$ were classified as completely masked, whereas 37 teeth (77.1\%) were classified as partially masked and no tooth remained unchanged. For partially masked teeth, the ratio of the white spot lesion to the whole tooth decreased significantly after treatment from $31.37 \%$ to $7.99 \%$. The surface color of infiltrated lesions remained stable after 12 months. ${ }^{(46)}$

Feng et al. $(2013)^{(47)}$ evaluated the effect of resin infiltration on masking white spot lesions by assessing the change in the white spot area in 74 maxillary anterior teeth with post-orthodontic decalcification using standardized digital images. Among the 74 teeth, 20 (27\%) were classified as completely masked and $54(73 \%)$ teeth were classified as partially masked. The ratio of the lesions to the whole tooth facial surface decreased from $39.28 \%$ to $9.46 \%$ after the treatment. ${ }^{(47)}$

Although researchers recommended the use of resin-infiltration technique for management of white spot lesions immediately following debonding of orthodontic appliances, successful results were obtained with late infiltration ${ }^{(48)}$ even after 8 months of debonding ${ }^{(20)}$ but there was need to re-etch the surface several times to remove the mineralized surface area. Kim et al. $(2011)^{(28)}$ recommended the use of resin infiltration technique after 3 months of debonding because after 3 months with careful oral hygiene, the active white spot lesion decreases spontaneously because of the remineralization by oral fluids. Some white spot lesions disappear within several weeks, and most lesions decrease with time until 3 months. ${ }^{(28,49,50)}$

The surfaces treated with resin infiltration technique were found to have an increased surface micro hardness than smooth surface initial caries lesions, ${ }^{\left({ }^{30)}\right.}$ increased resistance to further demineralization and mineral loss, ${ }^{(30)}$ and increased resistance to brushing abrasion than normal enamel. ${ }^{(51)}$ In addition, adhesion to such surfaces was similar to that of sound and demineralized enamel and even enhanced in some cases. ${ }^{(52,53)}$

Furthermore, proper planning for the management of white spot lesions should be made and assessment for the need of bleaching should be determined ahead as demineralized enamel treated with resin infiltration technique is less permeable to bleaching products as $10 \%$ hydrogen peroxide, thus producing no color alteration as demonstrated in vitro by Santos et al.(2012). ${ }^{(54)}$

\section{Conclusions}

The early detection of caries and the assessment of individual caries risk would be of a lot of help in preserving 
the tooth structure and avoiding unnecessary trauma to the dental tissues. When indicated, minimal invasive dentistry such as resin infiltration technique seems to provide a good solution in dealing with early enamel lesions as recommended by many recent studies in this field. Such technique could also be used in combination with other enamel remineralizing agents like fluoride varnishes, fluoride gels, non-sugar containing chewing gums......etc

Patient's motivation would probably play a major role in the success of any minimal invasive dentistry technique. It is always possible to shift to more invasive restorative procedures in case of failure of resin infiltration in patients where white spot lesions continue to progress and start to cavitate.

Further studies with longer periods of follow up are necessary to confirm the efficiency of this treatment modality and encourage the clinicians to use it in their dental practice.

\section{REFERENCES}

[1] Ericson D, Kidd E, McComb D, Mjör I, Noack MJ. Minimally Invasive Dentistry, concepts and techniques in cariology. Oral Health Prev Dent. 2003;1(1):59-72.

[2] Elizari JIZ. Minimal Intervention in Cariology - new devices for MI restorations. J Minim Interv Dent. 2011;4(3).

[3] Xie-Qi Shi, Sofia Tranæus, Birgit Angmar-Ma ${ }^{\circ}$ nsson. Validation of DIAGNOdent for quantification of smooth-surface caries: an in vitro study. Acta Odontol Scand. 2001:59(2):74-8.

[4] Kielbassa AM, Muller J, Gernhardt CR. Closing the gap between oral hygiene and minimally invasive dentistry: a review on the resin infiltration technique of incipient (proximal) enamel lesions. Quintessence international. 2009;40(8):663-81.

[5] Martignon S, Ekstrand KR, Ellwood R. Efficacy of sealing proximal early active lesions: an 18-month clinical study evaluated by conventional and subtraction radiography. Caries research. 2006;40(5):382-8.

[6] Phark JH, Duarte S, Jr., Meyer-Lueckel H, Paris S. Caries infiltration with resins: a novel treatment option for interproximal caries. Compendium of continuing education in dentistry. 2009;30(3):13-7.

[7] Paris S, Bitter K, Naumann M, Dorfer CE, Meyer-Lueckel H. Resin infiltration of proximal caries lesions differing in ICDAS codes. European journal of oral sciences. 2011;119(2):182-6.

[8] Weisrock G, Terrer E, Couderc G, Koubi S, Levallois B, Manton D, Tassery H: Naturally aesthetic restorations and minimally invasive dentistry. J Minim Interv Dent. 2011;4(2):23-30.

[9] Kugel G, Arsenault P, Papas A. Treatment modalities for caries management, including a new resin infiltration system. Compendium of continuing education in dentistry.2009;30(3):1-10.

[10] Meyer-Lueckel H, Paris S. Progression of artificial enamel caries lesions after infiltration with experimental light curing resins. Caries research. 2008;42(2):117-24.
[11] Meyer-Lueckel H, Paris S, Kielbassa AM. Surface layer erosion of natural caries lesions with phosphoric and hydrochloric acid gels in preparation for resin infiltration. Caries research. 2007;41(3):223-30.

[12] Paris S, Meyer-Lueckel H, Kielbassa AM. Resin infiltration of natural caries lesions. Journal of dental research. 2007;86(7):662-6.

[13] Meyer-Lueckel H, Paris S. Improved resin infiltration of natural caries lesions. Journal of dental research. 2008;87(12):1112-6.

[14] Paris S, Dorfer CE, Meyer-Lueckel H. Surface conditioning of natural enamel caries lesions in deciduous teeth in preparation for resin infiltration. Journal of dentistry. 2010;38(1):65-71.

[15] Meyer-Lueckel H, Paris S. Infiltration of natural caries lesions with experimental resins differing in penetration coefficients and ethanol addition. Caries research. 2010;44(4):408-14.

[16] Altarabulsi MB, Alkilzy M, Splieth CH. Clinical applicability of resin infiltration for proximal caries. Quintessence international. 2013;44(2):97-104.

[17] Lasfargues JJ, Bonte E, Guerrieri A, Fezzani L. Minimal intervention dentistry: part 6. Caries inhibition by resin infiltration. British dental journal. 2013;214(2):53-9.

[18] Robinson C, Brookes SJ, Kirkham J, Wood SR, Shore RC. In vitro studies of the penetration of adhesive resins into artificial caries-like lesions. Caries research. 2001;35(2):136-41.

[19] Paris S, Soviero VM, Schuch M, Meyer-Lueckel H. Pretreatment of natural caries lesions affects penetration depth of infiltrants in vitro. Clinical oral investigations. 2013;17(9):2085-9.

[20] Basaran G, Veli I, Basaran EG. Non-Cavitated Approach for the Treatment of White Spot Lesions: A Case Report. International Dental Research. 2011;1(2):65-9.

[21] de Barros L, Apolonio FM, Loguercio AD, de Saboia V. Resin-dentin bonds of etch-and-rinse adhesives to alcohol-saturated acid-etched dentin. The journal of adhesive dentistry. 2013;15(4):333-40.

[22] Li F, Liu XY, Zhang L, Kang JJ, Chen JH. Ethanol-wet bonding technique may enhance the bonding performance of contemporary etch-and-rinse dental adhesives. The journal of adhesive dentistry. 2012;14(2):113-20.

[23] Paris S, Meyer-Lueckel H. Masking of labial enamel white spot lesions by resin infiltration-a clinical report. Quintessence international. 2009;40(9):713-8.

[24] Liu Y, Ge L, Chen H, Chi X. A study on the penetration abilities of natural initial caries lesions with resin infiltration. West China journal of stomatology. 2012;30(5):483-6.

[25] Paris S, Lausch J, Selje T, Dorfer CE, Meyer-Lueckel H. Comparison of sealant and infiltrant penetration into pit and fissure caries lesions in vitro. Journal of dentistry. 2014;42(4):432-8.

[26] Paris S, Meyer-Lueckel H, Colfen H, Kielbassa AM. Resin infiltration of artificial enamel caries lesions with experimental light curing resins. Dental materials journal. 2007;26(4):582-8.

[27] Paris S, Schwendicke F, Keltsch J, Dorfer C, Meyer-Lueckel $\mathrm{H}$. Masking of white spot lesions by resin infiltration in vitro. Journal of dentistry. 2013;41(5):28-34.

[28] Kim S, Kim EY, Jeong TS, Kim JW. The evaluation of resin infiltration for masking labial enamel white spot lesions. International journal of paediatric dentistry. 
2011;21(4):241-8.

[29] Meyer-Lueckel H, Paris S, Mueller J, Colfen H, Kielbassa AM. Influence of the application time on the penetration of different dental adhesives and a fissure sealant into artificial subsurface lesions in bovine enamel. Dental materials. 2006;22(1):22-8.

[30] Paris S, Schwendicke F, Seddig S, Muller WD, Dorfer C, Meyer-Lueckel H. Micro-hardness and mineral loss of enamel lesions after infiltration with various resins: influence of infiltrant composition and application frequency in vitro. Journal of dentistry. 2013;41(6):543-8.

[31] Mueller J, Yang F, Neumann K, Kielbassa AM. Surface tridimensional topography analysis of materials and finishing procedures after resinous infiltration of subsurface bovine enamel lesions. Quintessence international. 2011;42(2):135-47.

[32] Paris S, Meyer-Lueckel H, Mueller J, Hummel M, Kielbassa AM. Progression of sealed initial bovine enamel lesions under demineralizing conditions in vitro. Caries research. 2006;40(2):124-9.

[33] Paris S, Meyer-Lueckel H. Inhibition of caries progression by resin infiltration in situ. Caries research. 2010;44(1):47-54.

[34] Paris S, Hopfenmuller W, Meyer-Lueckel H. Resin infiltration of caries lesions: an efficacy randomized trial. Journal of dental research. 2010;89(8):823-6.

[35] Martignon S, Ekstrand KR, Gomez J, Lara JS, Cortes A. Infiltrating/sealing proximal caries lesions: a 3-year randomized clinical trial. Journal of dental research. 2012;91(3):288-92.

[36] Vanderas AP, Manetas C, Koulatzidou M, Papagiannoulis L. Progression of proximal caries in the mixed dentition: a 4-year prospective study. Pediatric dentistry. $2003 ; 25(3): 229-34$.

[37] Vanderas AP, Gizani S, Papagiannoulis L. Progression of proximal caries in children with different caries indices: a 4-year radiographic study. European archives of paediatric dentistry. 2006;7(3):148-52.

[38] Skaleric U, Ravnik C, Cevc P, Schara M. Microcrystal arrangement in human deciduous dental enamel studied by electron paramagnetic resonance. Caries research. 1982;16(1):47-50.

[39] Paris S, Soviero VM, Seddig S, Meyer-Lueckel H. Penetration depths of an infiltrant into proximal caries lesions in primary molars after different application times in vitro. International journal of paediatric dentistry. 2012;22(5):349-55.

[40] Soviero VM, Paris S, Leal SC, Azevedo RB, Meyer-Lueckel H. Ex vivo evaluation of caries infiltration after different application times in primary molars. Caries research. 2013;47(2):110-6.

[41] Meyer-Lueckel H, Chatzidakis A, Naumann M, Dorfer CE, Paris S. Influence of application time on penetration of an infiltrant into natural enamel caries. Journal of dentistry. 2011;39(7):465-9.
[42] Ekstrand KR, Bakhshandeh A, Martignon S. Treatment of proximal superficial caries lesions on primary molar teeth with resin infiltration and fluoride varnish versus fluoride varnish only: efficacy after 1 year. Caries research. 2010;44(1):41-6.

[43] Gugnani N, Pandit IK, Gupta M, Josan R. Caries infiltration of noncavitated white spot lesions: A novel approach for immediate esthetic improvement. Contemporary clinical dentistry. 2012;3(Suppl 2):S199-202.

[44] Torres CR, Rosa PC, Ferreira NS, Borges AB. Effect of caries infiltration technique and fluoride therapy on microhardness of enamel carious lesions. Operative dentistry. 2012;37(4):363-9.

[45] Shivanna V, Shivakumar B. Novel treatment of white spot lesions: A report of two cases. Journal of conservative dentistry. 2011;14(4):423-6.

[46] Feng CH, Chu XY. Efficacy of one year treatment of icon infiltration resin on post-orthodontic white spots. Journal of Peking University Health sciences. 2013;45(1):40-3.

[47] Feng C, Liu R, Liu R, Zhao Q, Chu X. Effect of infiltration resin on the color masking of labial enamel white spot lesions. West China journal of stomatology. 2013;31(6):597-9.

[48] Neuhaus KW, Schlafer S, Lussi A, Nyvad B. Infiltration of natural caries lesions in relation to their activity status and acid pretreatment in vitro. Caries research. 2013;47(3):203-10.

[49] Artun J, Thylstrup A. Clinical and scanning electron microscopic study of surface changes of incipient caries lesions after debonding. Scandinavian journal of dental research. 1986;94(3):193-201.

[50] Artun J, Thylstrup A. A 3-year clinical and SEM study of surface changes of carious enamel lesions after inactivation. American journal of orthodontics and dentofacial orthopedics. 1989;95(4):327-33.

[51] Ungureanu L, Leon A, Nicolae A, Gabriela C. Micro-Invasive Treatment Of Non-Cavitated Carious Lesions In The Smooth Surfaces Of Teeth. International Journal of Medical Dentistry. 2012;2(1):11-6.

[52] Jia L, Stawarczyk B, Schmidlin PR, Attin T, Wiegand A. Effect of Caries Infiltrant Application on Shear Bond Strength of Different Adhesive Systems to Sound and Demineralized Enamel. Journal of Adhesive Dentistry. 2012;14(6):569.

[53] Yetkiner E, Özcan M, Wegehaupt FJ, Wiegand A, Eden E, Attin T. Effect of a Low-viscosity Adhesive Resin on the Adhesion of Metal Brackets to Enamel Etched with Hydrochloric or Phosphoric Acid Combined with Conventional Adhesives. Journal of Adhesive Dentistry. 2013;15(6):575.

[54] Thives de Freitas Santos LF, Chagas Rêgo HM, Bühler Borges A, Pucci CR, Rocha Gomes Torres C. Efficacy of Bleaching Treatment on Demineralized Enamel Treated with Resin Infiltration Technique. World Journal of Dentistry. 2012;3(4):279 